\title{
Compensating Acoustic Mismatch Using Class-Based Histogram Equalization for Robust Speech Recognition
}

\author{
Youngjoo Suh, Sungtak Kim, and Hoirin Kim \\ School of Engineering, Information and Communications University, 119 Munjiro, Daejeon 305-732, Yuseong-Gu, South Korea
}

Received 1 February 2006; Revised 26 November 2006; Accepted 1 February 2007

Recommended by Mark Gales

A new class-based histogram equalization method is proposed for robust speech recognition. The proposed method aims at not only compensating for an acoustic mismatch between training and test environments but also reducing the two fundamental limitations of the conventional histogram equalization method, the discrepancy between the phonetic distributions of training and test speech data, and the nonmonotonic transformation caused by the acoustic mismatch. The algorithm employs multiple class-specific reference and test cumulative distribution functions, classifies noisy test features into their corresponding classes, and equalizes the features by using their corresponding class reference and test distributions. The minimum mean-square error log-spectral amplitude (MMSE-LSA)-based speech enhancement is added just prior to the baseline feature extraction to reduce the corruption by additive noise. The experiments on the Aurora2 database proved the effectiveness of the proposed method by reducing relative errors by $62 \%$ over the mel-cepstral-based features and by $23 \%$ over the conventional histogram equalization method, respectively.

Copyright ( $) 2007$ Youngjoo Suh et al. This is an open access article distributed under the Creative Commons Attribution License, which permits unrestricted use, distribution, and reproduction in any medium, provided the original work is properly cited.

\section{INTRODUCTION}

The performance of automatic speech recognition (ASR) systems degrades severely when they are employed in acoustically mismatched environments compared to the training ones. The main cause of this acoustic mismatch is corruption by additive noise and channel distortion, both of which are commonly encountered adverse sources in the real-world ASR applications. To cope with this problem, robust speech recognition has become one of the most crucial issues in the research area of speech recognition. Currently, most robust speech recognition methods can be categorized into the following three areas: signal space, feature space, and model space [1]. Compared to the other two categories, the feature space approach has also been widely employed due to advantages such as easy implementation, low computational complexity, and effective performance improvements. Acoustic environments corrupted by additive noise and channel distortion act as a nonlinear transformation in the feature spaces of the cepstrum or log-spectrum [2]. Thus, classical linear feature space methods such as cepstral mean subtraction or cepstral mean and variance normalization have substantial limitations even though they yield significant performance improvements under noisy environments [3-5]. Currently, the major feature space approaches to reducing the nonlinear behaviors of the acoustic mismatch are based on the piecewise linear approximation, such as interacting multiple model (IMM) [6] and stereo-based piecewise linear compensation for environments (SPLICE) [7]. Another effective environmental compensation method that transforms observed features is constrained maximum likelihood linear regression (CMLLR) although it is not strictly based on the feature space. In the related literature [8], its performance was shown to be comparable to those of other linear model space transformation methods. However, like other model space transformation methods, CMLLR also requires at least several speech utterances for its reliable estimation of the transformation matrix and it is still classified as a linear transform-based approach.

As an alternative approach to coping with the drawbacks of linear transform-based methods, the histogram equalization (HEQ) technique has been employed to compensate for the acoustic mismatch. While HEQ was originally introduced to image processing applications [9], recent research has shown that it is also quite effective in preventing performance degradation in ASR under noisy environments 
[10-17]. Moreover, in contrast with most linear transformbased approaches, HEQ is computationally more efficient because its algorithm mostly consists of sorting and search (or table look-up) routines. The role of HEQ is to transform test features to reference ones in order to compensate for the acoustic mismatch between the training and test environments by converting the probability density function (PDF) of the original test variable into its reference (or training) PDF. In order to compensate for the acoustic mismatch more effectively, HEQ has two fundamental requirements. First, distributions of phonetic or acoustic classes, defined in the acoustic modeling of speech recognition systems, for both training and test data should be identical or similar to each other [18]. Second, acoustic mismatch should act as a monotonic transformation in the feature space [17]. In other words, the ordering information of phonetic or acoustic classes along each feature axis should not be altered by the acoustic mismatch. When these requirements are not kept, the ordering information of phonetic or acoustic classes in features can be changed by the acoustic mismatch and as a result, the transformation by HEQ can impair class separability of the features. However, in most speech recognition applications, test speech utterances tend to be too short to make their phonetic or acoustic class distributions identical or similar to those of training data. Furthermore, corruption by additive noise or channel distortion is considered as a random transformation in the feature space. This random behavior does not always guarantee the monotonic transformation. Therefore, the above-mentioned requirements are not generally satisfied in real-world speech recognition applications. As a result, it is difficult to take full advantage of HEQ when the conventional HEQ is used to compensate for the acoustic mismatch in noisy environments.

In this paper, we propose a new class-based HEQ technique to reduce these two limitations of the conventional HEQ method. Instead of utilizing global reference and test cumulative distribution functions (CDFs) as in the conventional HEQ, the proposed method employs multiple classbased CDFs not only to compensate for the acoustic mismatch between training and test data but also to reduce the limitations of the conventional HEQ. Based on the fact that HEQ is not able to compensate for the adverse effect caused by temporally random behavior of noise, we also introduce the minimum mean-square error log-spectral amplitude (MMSE-LSA)-based speech enhancement technique [19] that is used as a front-end preprocessor to HEQ to further reduce the acoustic mismatch.

The rest of this paper is organized as follows. Section 2 provides a brief review of the MMSE-LSA-based speech enhancement algorithm used in this work. Section 3 describes the basic algorithm of the conventional HEQ. In Section 4, we present the proposed class-based HEQ technique that reduces the two limitations of the conventional HEQ for compensating for the acoustic mismatch in speech recognition under noisy environments. Section 5 describes experimental results of our proposed method. Finally, concluding remarks will be given in Section 6 .

\section{SPEECH ENHANCEMENT BASED ON MMSE-LSA}

HEQ utilizes CDFs of both reference and test data to compensate for the acoustic mismatch. Therefore, this method does not take into account specific temporal characteristics of noise but deals with the property of how long-term distributions of noisy speech representations differ from those of clean reference speech ones. Thus, it focuses more on speech than noise in the compensation of the acoustic mismatch. On the contrary, most speech enhancement methods reduce noise components from noisy speech representations by firstly estimating noise characteristics such as noise power or magnitude spectra. In this case, random behaviors of noise are regarded more importantly. From these different approaches, we expect that the use of a proper speech enhancement technique in combination with HEQ will provide additional compensation effects than that of HEQ alone. In this paper, we employ the MMSE-LSA algorithm as a frontend speech enhancement method that is used prior to the feature extraction to additionally compensate for the acoustic mismatch. A brief review of the MMSE-LSA algorithm is given as follows [19-21].

Let $S_{k}(n)=A_{k}(n) e^{j \varphi_{k}(n)}, D_{k}(n)$, and $U_{k}(n)=R_{k}(n) e^{j \vartheta_{k}(n)}$ be the frequency components of clean speech $s(t)$, additive noise $d(t)$, and noisy speech $u(t)$ at frequency bin index $k$, time frame index $n$, and time sample index $t$, respectively. When $S_{k}(n)$ and $D_{k}(n)$ are assumed to be characterized by separate zero-mean complex Gaussian distributions, the MMSE-LSA estimate of a clean speech spectrum, $\hat{A}_{k}(n)$, is obtained by the estimation criterion that minimizes the mean-square error of log-spectral amplitude for given noisy spectrum $U_{k}(n)$ and is given by

$$
\widehat{A}_{k}(n)=\frac{\Lambda_{k}(n)}{1+\Lambda_{k}(n)} G_{\mathrm{MMSE}-\mathrm{LSA}, k}(n) R_{k}(n)
$$

where $G_{\mathrm{MMSE}-\mathrm{LSA}, k}(n)$ is derived as

$$
G_{\mathrm{MMSE}-\mathrm{LSA}, k}(n)=\frac{\xi_{k}(n)}{1+\xi_{k}(n)} \exp \left(\frac{1}{2} \int_{\nu_{k}(n)}^{\infty} \frac{e^{-\tau}}{\tau} d \tau\right)
$$

where $\nu_{k}(n)=\left(\xi_{k}(n) /\left(1+\xi_{k}(n)\right)\right) \gamma_{k}(n), \gamma_{k}(n)=R_{k}^{2}(n) / \lambda_{d, k}(n)$, $\xi_{k}(n)=\eta_{k}(n) /\left(1-q_{k}(n)\right), \eta_{k}(n)=\lambda_{s, k}(n) / \lambda_{d, k}(n), \lambda_{s, k}(n)=$ $E\left\{\left|S_{k}(n)\right|^{2}\right\}=E\left\{A_{k}^{2}(n)\right\}$, and $\lambda_{d, k}(n)=E\left\{\left|D_{k}(n)\right|^{2}\right\} . \eta_{k}(n)$ and $\gamma_{k}(n)$ are called a priori and a posteriori signal-to-noise ratios (SNR), respectively. $q_{k}(n)$ is called the a priori probability of speech absence and is fixed to 0.2 for all frequency bins and time frames in this paper. $\lambda_{s, k}(n)$ and $\lambda_{d, k}(n)$ denote power spectral densities of speech and noise, respectively.

The likelihood ratio between speech presence and absence, $\Lambda_{k}(n)$, is defined by

$$
\Lambda_{k}(n)=\left.\frac{1-q_{k}(n)}{q_{k}(n)} \frac{\exp \left(\nu_{k}(n)\right)}{1+\xi_{k}(n)}\right|_{\xi_{k}(n)=\eta_{k}(n) /\left(1-q_{k}(n)\right)} .
$$


In our experiments, $\lambda_{d, k}(n)$ is estimated by the mixed decision-based decision-directed approach [22-24] given by

$$
\begin{aligned}
& \lambda_{d, k}(n+1)
\end{aligned}
$$

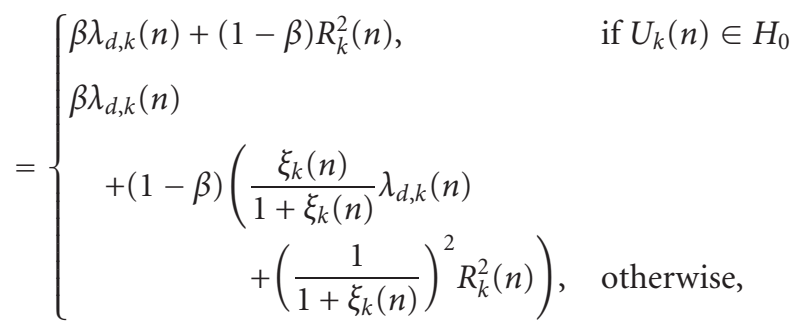

where $H_{0}$ is the speech absence hypothesis that is usually determined by a voice activity detector and $\beta$ is a forgetting factor empirically chosen as 0.98 .

When the gain function of the estimator is aggressively estimated, enhanced speech signals tend to suffer from signal distortion [25]. On the other hand, in case of underestimation, they contain a significant amount of residual noise. Thus, the degree of aggression needs to be chosen carefully to obtain the maximum gain in the sense of speech recognition accuracy. The method to determine the degree of aggression in these experiments is similar to that used in the Aurora advanced front-end noise reduction algorithm [25] except that an empirically chosen fixed value is used in this case.

\section{CONVENTIONAL HISTOGRAM EQUALIZATION}

Currently, there are two approaches to matching reference and test CDFs in the HEQ-based feature space transformation. The first one is the use of empirical CDFs and the other is the adoption of Gaussianization [26, 27]. Although the former approach requires far more parameters and their adaptation data, its main advantages are that (1) it can bypass the problems associated with choosing the size of the mixture models [27] and (2) it is considered as a nonparametric method which does not require any specific assumptions about the probability distribution of modeling data. On the contrary, one merit of the latter approach is that Gaussianization of the features can enforce the modeling assumption in the HMM-based ASR where the output probabilities are modeled with mixtures of diagonal covariance Gaussians. Here, the main focus of our approach is on the use of multiple classes in the nonlinear feature space transformation. Therefore, we only deal with HEQ utilizing empirical CDFs for CDF matching in this paper and its detailed descriptions are given as follows.

For given random reference and test variables $x$ and $y$ whose corresponding PDFs are given as $P_{X}(x)$ and $P_{Y}(y)$, respectively, a transform function $x=F(y)$ mapping $P_{Y}(y)$ into $P_{X}(x)$ can be given as $[9,17]$

$$
x=F(y)=C_{X}^{-1}\left(C_{Y}(y)\right),
$$

where $C_{X}^{-1}(x)$ is the inverse of reference $\operatorname{CDF} C_{X}(x)$ and $C_{Y}(y)$ is the test CDF of random variable $y$, respectively.
Of course, most current speech recognition algorithms utilize multidimensional feature vectors as their feature parameters, where each feature vector consists of a number of coefficients. When the feature parameters are transformed on a multidimensional vector basis, HEQ requires the joint CDF transformation involving Jacobian operations. However, the joint CDF transformation is generally a difficult problem as in [26]. Thus, we make a simplified assumption that the feature coefficients are statistically independent of each other [27]. This assumption is especially acceptable when decorrelated filter-bank log-energies [28] or cepstral coefficients are used as recognition features because of their low degree of cross-correlation. Therefore, for the sake of algorithmic simplicity, we only deal with the CDF transformation on a component-by-component basis in this paper.

Another critical issue in HEQ is the reliable estimation of reference and test CDFs. In speech recognition applications, the amount of training data is usually large. Thus, reference CDFs can be estimated quite reliably by computing cumulative histograms using training data. However, when short utterances are used as test data, the lengths of such utterances may be insufficient for a reliable estimation. In these test environments, the test CDF estimation becomes much more important. When the amount of estimation samples is small, the order-statistic-based CDF estimation is preferred rather than the cumulative histogram-based method and its brief description is as follows $[12,16]$.

Let us define a sequence consisting of $N$ frames of a particular feature component as

$$
V_{l}=\left\{y_{l}(1), y_{l}(2), \ldots, y_{l}(n), \ldots, y_{l}(N)\right\}
$$

where $y_{l}(n)$ denotes the $l$ th feature component at the $n$th frame.

The order statistics of (6) can be defined as

$$
y_{l}([1]) \leq \cdots \leq y_{l}\left(\left[r_{l}\right]\right) \leq \cdots \leq y_{l}([N]),
$$

where $\left[r_{l}\right]$ represents the original frame index of the feature component $y\left(\left[r_{l}\right]\right)$ at which its rank is denoted as $r_{l}$ when the elements of the sequence $V_{l}$ are sorted in ascending order.

Then, given test feature component $y_{l}(n)$, the orderstatistic-based direct estimate of test CDFs can be defined as

$$
\hat{C}_{Y(l)}\left(y_{l}(n)\right)=\frac{R_{l}\left(y_{l}(n)\right)-0.5}{N}, \quad 1 \leq l \leq L,
$$

where $R_{l}\left(y_{l}(n)\right)$ denotes the rank of $y_{l}(n)$ ranging from 1 to $N$ and $L$ stands for the total dimension of the feature vector.

An estimate of the reference feature component by the conventional HEQ given test feature component $y_{l}(n)$ is obtained as

$$
\hat{x}_{l}(n)=C_{X(l)}^{-1}\left(\hat{C}_{Y(l)}\left(y_{l}(n)\right)\right)=C_{X(l)}^{-1}\left(\frac{R_{l}\left(y_{l}(n)\right)-0.5}{N}\right) \text {. }
$$

According to the adoption of empirical CDFs in CDF matching in this paper, all reference CDFs are modeled by using cumulative histograms. Moreover, the transformation by the inverse of each reference CDF in (9) is performed with a linear interpolation by taking into account the relative position within the histogram bin to reduce the mapping error [17]. 


\section{CLASS-BASED HISTOGRAM EQUALIZATION}

\subsection{Basic algorithm}

The proposed approach for reducing both the acoustic mismatch and the limitations of the conventional HEQ consists of utilizing multiple class-specific CDFs at both reference and test sides. To solve these two problems, it divides global distributions defined in the conventional HEQ into sets of multiple class distributions, classifies feature components into their classes, and then transforms them using their corresponding class CDFs [18]. By this approach, the mismatch of phonetic class distributions can be effectively reduced because of the increased similarity between the reference and test distributions within the same class. In addition, the global-level nonmonotonic transformation, the second limitation of the conventional HEQ, can be restricted only to a class level only if class information is reliably assigned to each feature coefficient. However, reliably assigning class information to each feature component is a prerequisite condition for ensuring the validity of the proposed HEQ method. In most HEQ methods, the equalization is performed on a component-by-component basis for the sake of algorithmic simplicity as well as reliable CDF estimation. In this sense, the phonetic classification can be also performed on a feature component basis. However, utilizing a feature vector instead of only a specific feature component is more useful in phonetic classification and thus employed in the proposed method. Nevertheless, it may be still a critical problem to accurately classify feature vectors into their corresponding phonetic classes in noisy environments. To cope with such a problem, we use a histogram equalized feature vector in the classification instead of the original noisy feature vector to reduce the adverse effects by additive noise and channel distortion. A detailed description of the proposed class-based HEQ is given as follows.

Let us define a noisy feature vector $W_{n}$ consisting of $L$ dimensional components at time frame $n$ as

$$
W_{n}=\left\{y_{1}(n), y_{2}(n), \ldots, y_{L}(n)\right\}^{T}
$$

where $T$ stands for vector transpose.

Then, phonetic class index $i$ assigned to noisy feature vector $W_{n}$ is obtained as

$$
\widehat{i}=\arg \min _{i} d\left(\widehat{W}_{n}, z_{i}\right), \quad 1 \leq i \leq I
$$

where $d(\cdot, \cdot)$ denotes the Mahalanobis distance measure, $z_{i}$ stands for the centroid of the $i$ th class computed by the $k$ means algorithm, $I$ is the number of classes, and $\widehat{W}_{n}$ is the histogram equalized version of $W_{n}$ by the conventional HEQ given as follows:

$$
\begin{aligned}
\widehat{W}_{n} & =\left\{\hat{x}_{1}(n), \ldots, \hat{x}_{L}(n)\right\}^{T} \\
& =\left\{C_{X(1)}^{-1}\left(\widehat{C}_{Y(1)}\left(y_{1}(n)\right)\right), \ldots, C_{X(L)}^{-1}\left(\widehat{C}_{Y(L)}\left(y_{L}(n)\right)\right)\right\}^{T} .
\end{aligned}
$$

\subsection{Class-tying technique}

According to the basic idea of the class-based HEQ, the limitations of the conventional HEQ can be effectively reduced by increasing the number of phonetic classes to a sufficient level, only if the phonetic classification accuracy is sufficiently high. However, the phonetic classification accuracy tends to be inevitably decreased in noisy environments. In such noisy conditions, increasing the number of phonetic classes further deteriorates the classification accuracy due to increased class candidates. At the same time, increasing the number of phonetic classes also decreases the amount of classified sample data for each phonetic class, which deteriorates the reliability of test CDF estimation. For these reasons, the performance of the class-based HEQ increases to a certain number of phonetic classes, and then tends to decrease. As a result, we cannot increase the number of phonetic classes arbitrarily to keep the classification accuracy within an allowable level and, at the same time, provide more reliable test CDF estimation. To provide higher phonetic classification accuracy as well as more reliable test CDF estimation, the class-tying technique is employed so that a number of small similar phonetic untied classes are tied into a larger tied class. The tying rule between small untied classes and a single larger tied class is determined such that the tied class $\hat{j}$ for a certain small untied class $\hat{i}$ is obtained by

$$
\hat{j}=\arg \min _{j} d\left(z_{\hat{i}}, Z_{j}\right), \quad 1 \leq j \leq J
$$

where $Z_{j}$ represents the centroid of the $j$ th tied class, each of which is computed by using vector quantization, where all centroids of the small untied classes defined in (11) are used as training sample data. In addition, $J$ (where $J<I$ ) is the number of tied classes.

Then, the proposed class-based HEQ formulation for given test feature component $y_{l}(n)$ is defined as

$\tilde{x}_{l}(n)=C_{X(\hat{j}, l)}^{-1}\left(C_{Y(\hat{j}, l)}\left(y_{l}(n)\right)\right)=C_{X(\hat{j}, l)}^{-1}\left(\frac{R_{\hat{j}, l}\left(y_{l}(n)\right)-0.5}{N_{\hat{j}}}\right)$,

where, $C_{Y(\hat{j}, l)}(y)$ and $R_{\hat{j}, l}(y)$ denote the test CDF and the rank at the $\hat{j}$ th tied class and $l$ th feature component, respectively, $N_{\hat{j}}$ is the number of frames which are classified as the $\hat{j}$ th tied class, and $C_{X(\hat{j}, l)}^{-1}(x)$ represents the inverse of reference $\operatorname{CDF} C_{X(\hat{j}, l)}(x)$ which is obtained by the cumulative histogram computed from all training data of the $l$ th feature components which are classified as the $\hat{j}$ th tied class by the vector quantization-based phonetic classification.

In exceptional cases, where the number of frames classified into a particular tied class is less than the threshold value (in our case, empirically chosen as 5), the equalization is performed by the conventional HEQ for more reliable CDF estimation. Figure 1 shows the overall structure of the proposed compensation method where the MMSE-LSA-based speech enhancement algorithm is optionally added as a front-end to the feature extraction. In this figure, the global HEQ refers to 


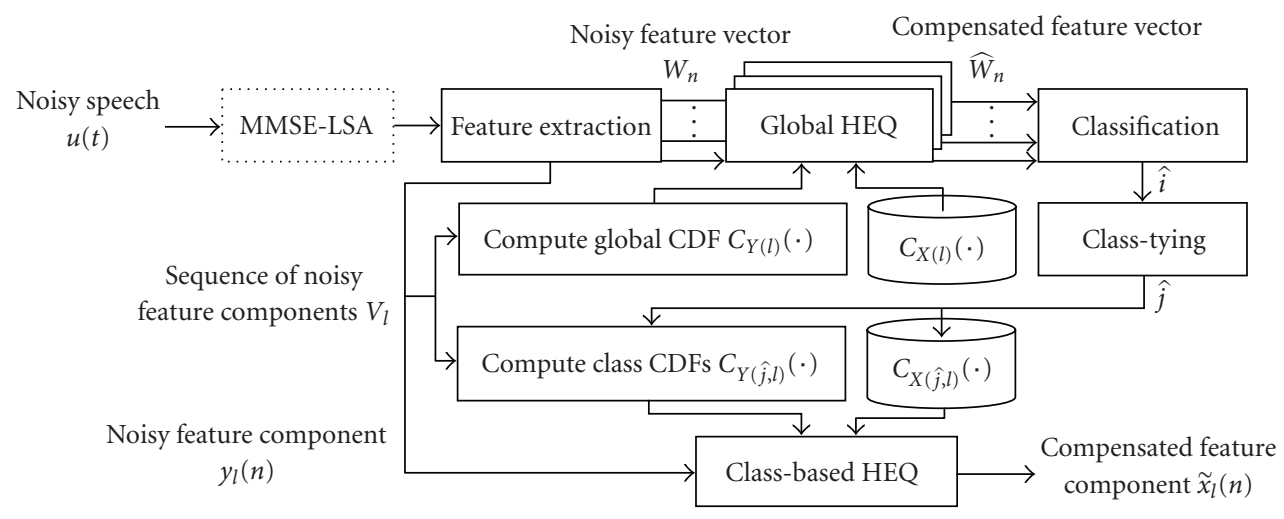

FIGURE 1: Block diagram of the proposed acoustic mismatch compensation method based on the class-based HEQ with the MMSE-LSAbased speech enhancement.

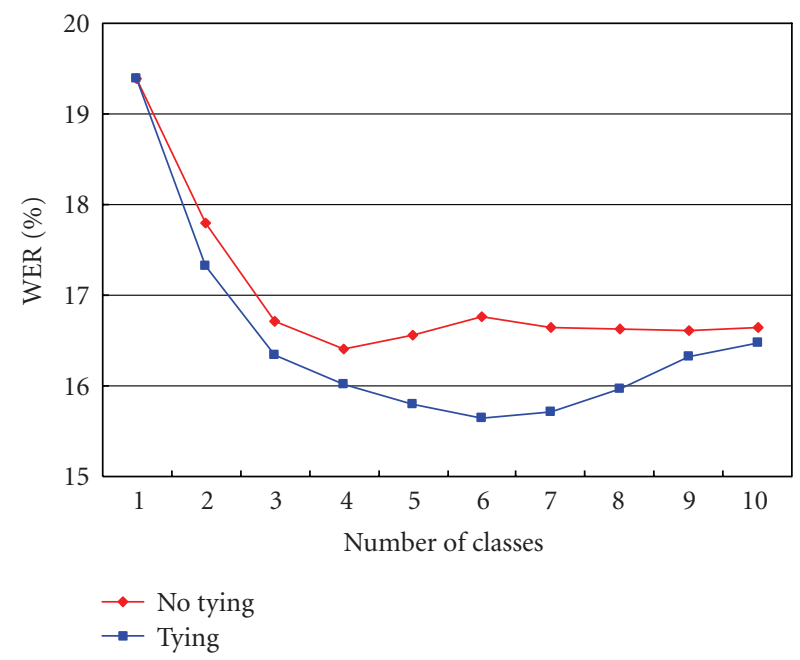

FIgURE 2: Recognition results of untied/tied class CHEQ compensation techniques with regard to various numbers of classes on the Aurora 2 task (clean-condition training).

the conventional HEQ from the fact that it uses global reference and test CDFs.

\section{EXPERIMENTAL RESULTS}

\subsection{Speech database and feature extraction}

In the performance evaluation, the Aurora2 database which is converted from the TI-DIGITS database is used. Only clean speech data are used in the training of all experiments (i.e., clean-condition training). Test sets $\mathrm{A}$ and $\mathrm{B}$, each containing four kinds of additive noises, and test set C, contaminated by two kinds of additive noises and different channel distortion (MIRS), are chosen in the test. The MMSE-LSA-based speech enhancement (SE) technique is applied at the signal space. In SE, a 25 millisecond long Hamming window is applied to noisy speech signals with an interval of 10 millisecond. FFT with 256 points is used for spectral analysis. Enhanced speech signals reconstructed by the overlap-add method are then used for feature extraction.

The feature extraction procedure is conducted based on the ETSI Aurora formula as follows. First, speech signals are blocked into a sequence of frames, each with 25 millisecond length and 10 millisecond interval. Next, speech frames are pre-emphasized with a factor of 0.97 and then a Hamming window is applied to each speech frame. From a set of 23 mel-scaled filter-bank log-energies, the 39-dimensional melfrequency cepstral coefficient (MFCC)-based feature vector consisting of 12 MFCCs, the log-energy, and their first and second derivatives is extracted. Prior to the derivative computations, the 22-order cepstral liftering is applied to the static MFCCs. Each digit-based hidden Markov model consists of 16 states and each state has 3 mixtures. The number of histogram bins in reference CDFs was chosen as 64 in both conventional HEQ (HEQ) and class-based HEQ (CHEQ) because its further increase did not show any meaningful performance improvements. The tied class parameters, $I$ and $J$, are empirically set to 60 and 6, respectively, based on the experimental results shown in Figure 2. The equalization is conducted on each component of the 39-dimensional MFCCs for both training and test data on an utterance-byutterance basis.

\subsection{Speech recognition results}

Figure 2 shows recognition results when the CHEQ method is used alone or in combination with the class-tying technique to compensate for the acoustic mismatch in noisy features. The results represent averaged values of word-error rate (WER) for the three test sets with respect to a number of tied classes ranging from one (i.e., conventional HEQ case) to ten. WERs are averaged between $0 \mathrm{~dB}$ and $20 \mathrm{~dB}$ SNR as recommended by the Aurora group. In the experiments of tied class cases, the corresponding untied classes are empirically chosen as those producing the lowest WER between 20 to 100 untied classes. In this figure, we observe that CHEQ provides significant improvements over HEQ only when the number of classes exceeds two. Above this 
TABLE 1: Average WERs (\%) of various acoustic compensation techniques on the Aurora2 task (clean-condition training, averaged between $0-20 \mathrm{~dB}$ SNRs).

\begin{tabular}{l|cccccc}
\hline Test data & Baseline & SE & HEQ & CHEQ & SE + HEQ & SE + CHEQ \\
\hline Set A & 38.88 & 22.61 & 19.41 & 15.77 & 15.11 & 17.81 \\
Set B & 44.43 & 26.97 & 18.32 & 16.50 & 17.21 & 14.38 \\
Set C & 33.32 & 26.84 & 21.55 & 15.65 & 18.10 & 16.11 \\
Average & 39.99 & 25.20 & 19.40 & 60.86 & 54.72 & 62.76 \\
ERR & - & 36.98 & 51.48 & & \\
\hline
\end{tabular}

number, the performance improvement seems marginal for the untied-CHEQ case. However, further improvements are still obtained for the tied-CHEQ case. From this figure, it is well observed that CHEQ is very effective in improving recognition performance compared to HEQ and the tied class technique provides an additional gain with a maximum error-rate reduction (ERR) of 4.65\%. However, as mentioned in Section 4.2, we also notice that the recognition accuracy tends to deteriorate for more phonetic classes than those producing the best performance, mainly due to the decreased phonetic classification accuracy in noisy environment.

Table 1 presents the recognition results obtained by using the baseline feature (i.e., MFCC) and compensation techniques each of which is applied alone or in combination with one of the other methods under clean-condition training. In the experiments, MMSE-LSA-based SE was applied only to test data while HEQ and CHEQ are applied to both training and test data. For all test sets, all of the three compensation methods reduce relative errors by more than $30 \%$ even in the case that each technique is used alone. It is also observed that HEQ is far more effective than SE. In addition, CHEQ offers substantial improvements over HEQ by an ERR of 19\%. Applying SE to HEQ and CHEQ produces slight improvements by ERRs of $6 \%$ and $4 \%$, respectively, indicating that MMSELSA-based SE and histogram equalization do not act as fully additive each other when they are used together in compensating for the acoustic mismatch. In this case, CHEQ with SE provides less improvement than HEQ with SE. This may be due to the fact that major causes of the nonmonotonic transformation can be effectively removed as the preprocessor SE reduces noise. The addition of SE produces substantial improvements for test sets A and B but offers marginal error reduction for test set $\mathrm{C}$. These results comply with the fact that the MMSE-LSA-based SE is only effective in reducing additive noise and has less capability of canceling the channel distortion. It is also noted that the performance improvements by HEQ-based compensation methods on test set $\mathrm{C}$ are less than those on test sets $\mathrm{A}$ and $\mathrm{B}$. These results mean that the compensation techniques are not as effective for acoustic environments suffering from both additive noise and channel distortion together as those containing additive noise only. Nevertheless, when we compare recognition results for CHEQ with those for SE and HEQ, we still observe that the degradation on test set $\mathrm{C}$ by CHEQ is much less than those by SE or HEQ. More complex acoustic environments including additive noise and channel distortion together tend to have higher possibilities for the nonmonotonic transfor- mation than those presenting only additive noise. In these acoustic environments, reduced degradation in recognition accuracy by CHEQ implies that the ability of CHEQ to reduce the nonmonotonic transformation is its discriminative superiority compared to HEQ.

Table 2 through Table 7 show detailed recognition results when the baseline MFCC feature or compensated features by various compensation techniques are used. It is observed that SE reduces errors moderately for all types of noises and different channel environments while slight degradations are found for the clean condition. However, large variations of average WERs for different noise types imply weak noise robustness of SE. On the contrary, HEQ provides larger error reduction than SE for the same kinds of noises and channel environments. It even reduces errors for the clean condition. Smaller variation of average WERs for different noise types in HEQ indicates its relative robustness over various noise conditions and confirms its merit that HEQ does not require any assumptions on noise characteristics. Finally, CHEQ offers the largest error reduction of all three compensation methods. It seems especially useful for the noise types of car, airport, street, and station but relatively less effective in those of babble, exhibition, and restaurant. The former noises are largely related to the engine noise, while the latter contain human speech-like noises considerably. We think the less effectiveness of CHEQ on the human speech-like noises is mainly a result of the lower phonetic classification accuracy at this category of noises. However, the relatively small variation of average WERs by CHEQ for different kinds of noises implies its invariant effectiveness over various noises. From Table 4 to Table 7, it is illustrated that CHEQ is much superior to HEQ for the SNR conditions lower than $20 \mathrm{~dB}$ but less effective for clean and $20 \mathrm{~dB}$ conditions. The presence of noise is less prominent at high SNR conditions. Thus, the nonmonotonic transformations by the acoustic mismatch at these high SNR conditions are expected to be less severe, which can reduce the room for further improvements by CHEQ. In addition, decreased reliability of the class-based test CDF estimation caused by a reduced amount of classified data could overweigh the gain from the use of a class concept in $\mathrm{CHEQ}$, and this seems to be another cause of performance degradation by CHEQ in these high SNR conditions. At the same time, the performance degradation by CHEQ in high SNR environments also strongly implies that the limitation of HEQ by the nonmonotonic transformation is much dominant than that by the mismatch of phonetic class distributions between training and test data. 
TABLE 2: Recognition results of the baseline feature (MFCC) on the Aurora2 task under clean-condition training (WER \%).

\begin{tabular}{l|rrrrr|rrrrrrrr}
\hline \multirow{2}{*}{ SNR } & \multicolumn{5}{|c|}{ Set A } & \multicolumn{5}{c}{ Set B } & \multicolumn{2}{c}{ Set C } \\
\cline { 2 - 15 } & Subway & Babble & Car & Exhib. & Avg. & Rest. & Street & Airport & Station & Avg. & Subway & Street & Avg. \\
\hline Clean & 1.17 & 1.03 & 1.19 & 0.86 & 1.06 & 1.17 & 1.03 & 1.19 & 0.86 & 1.54 & 0.98 & 1.03 & 1.01 \\
$20 \mathrm{~dB}$ & 3.04 & 10.04 & 3.16 & 3.80 & 5.01 & 10.81 & 4.23 & 9.93 & 5.62 & 3.50 & 5.53 & 4.81 & 5.17 \\
$15 \mathrm{~dB}$ & 7.09 & 26.57 & 10.47 & 8.15 & 13.07 & 25.61 & 11.73 & 23.11 & 16.38 & 5.00 & 12.37 & 10.31 & 11.34 \\
$10 \mathrm{~dB}$ & 21.28 & 50.94 & 33.76 & 24.90 & 32.72 & 47.28 & 33.25 & 46.85 & 40.39 & 9.21 & 24.81 & 24.73 & 24.77 \\
$5 \mathrm{~dB}$ & 46.61 & 72.97 & 66.51 & 56.49 & 60.65 & 70.43 & 61.85 & 69.31 & 70.26 & 20.51 & 47.16 & 51.15 & 49.16 \\
$0 \mathrm{~dB}$ & 72.70 & 88.27 & 86.73 & 84.02 & 82.93 & 88.30 & 81.32 & 84.16 & 87.75 & 44.70 & 73.99 & 78.36 & 76.18 \\
$-5 \mathrm{~dB}$ & 87.38 & 95.04 & 91.65 & 92.35 & 91.61 & 95.00 & 89.93 & 91.89 & 91.51 & 77.24 & 87.90 & 89.30 & 88.60 \\
Avg. & 30.14 & 49.76 & 40.13 & 35.47 & 38.88 & 48.49 & 38.48 & 46.67 & 44.08 & 44.43 & 32.77 & 33.87 & 33.32 \\
\hline
\end{tabular}

TABLE 3: Recognition results of the SE compensation technique on the Aurora 2 task under clean-condition training (WER \%).

\begin{tabular}{|c|c|c|c|c|c|c|c|c|c|c|c|c|c|}
\hline \multirow{2}{*}{ SNR } & \multicolumn{5}{|c|}{ Set A } & \multicolumn{5}{|c|}{ Set B } & \multicolumn{3}{|c|}{ Set C } \\
\hline & Subway & Babble & Car & Exhib. & Avg. & Rest. & Street & Airport & Station & Avg. & Subway & Street & Avg. \\
\hline Clean & 1.63 & 1.81 & 1.79 & 1.48 & 1.68 & 1.63 & 1.81 & 1.79 & 1.48 & 98.37 & 1.63 & 1.72 & 1.68 \\
\hline $20 \mathrm{~dB}$ & 2.86 & 3.11 & 2.33 & 4.38 & 3.92 & 3.84 & 2.90 & 3.55 & 3.18 & 96.16 & 4.21 & 3.66 & 3.94 \\
\hline $15 \mathrm{~dB}$ & 4.97 & 7.19 & 2.98 & 6.11 & 7.07 & 9.21 & 4.93 & 6.95 & 5.37 & 90.79 & 8.14 & 7.04 & 7.59 \\
\hline $10 \mathrm{~dB}$ & 9.58 & 17.74 & 6.98 & 11.82 & 14.87 & 20.85 & 10.43 & 17.30 & 11.88 & 79.15 & 18.67 & 16.90 & 17.79 \\
\hline $5 \mathrm{~dB}$ & 25.76 & 43.02 & 22.34 & 27.21 & 29.19 & 45.47 & 29.72 & 41.07 & 33.66 & 54.53 & 41.11 & 35.67 & 38.39 \\
\hline $0 \mathrm{~dB}$ & 55.97 & 78.45 & 62.51 & 56.87 & 54.18 & 78.32 & 64.45 & 74.74 & 71.52 & 21.68 & 70.00 & 63.03 & 66.52 \\
\hline$-5 \mathrm{~dB}$ & 82.71 & 95.80 & 89.08 & 85.75 & 79.08 & 96.84 & 86.61 & 92.93 & 91.89 & 3.16 & 85.82 & 83.92 & 84.87 \\
\hline Avg. & 19.83 & 29.90 & 19.43 & 21.28 & 22.61 & 31.54 & 22.49 & 28.72 & 25.12 & 26.97 & 28.43 & 25.26 & 26.84 \\
\hline
\end{tabular}

TABLE 4: Recognition results of the HEQ compensation technique on the Aurora 2 task under clean-condition training (WER \%).

\begin{tabular}{l|rrrrr|rrrrrrrr}
\hline \multirow{2}{*}{ SNR } & \multicolumn{5}{|c|}{ Set A } & \multicolumn{5}{c}{ Set B } & \multicolumn{2}{c}{ Set C } \\
\cline { 2 - 14 } & Subway & Babble & Car & Exhib. & Avg. & Rest. & Street & Airport & Station & Avg. & Subway & Street & Avg. \\
\hline Clean & 1.14 & 1.00 & 0.92 & 0.96 & 1.01 & 1.14 & 1.00 & 0.92 & 0.96 & 1.01 & 1.07 & 1.09 & 1.08 \\
$20 \mathrm{~dB}$ & 3.44 & 2.36 & 2.62 & 3.92 & 3.09 & 2.82 & 2.63 & 2.54 & 3.02 & 2.75 & 3.84 & 2.81 & 3.33 \\
$15 \mathrm{~dB}$ & 6.69 & 4.38 & 4.59 & 7.07 & 5.68 & 4.85 & 4.75 & 4.35 & 5.25 & 4.80 & 6.66 & 5.35 & 6.01 \\
$10 \mathrm{~dB}$ & 11.39 & 9.10 & 9.60 & 14.87 & 11.24 & 10.10 & 9.70 & 9.01 & 10.80 & 9.90 & 13.72 & 12.61 & 13.17 \\
$5 \mathrm{~dB}$ & 23.24 & 23.22 & 23.23 & 29.19 & 24.72 & 22.54 & 23.00 & 21.92 & 24.44 & 22.98 & 28.86 & 26.63 & 27.75 \\
$0 \mathrm{~dB}$ & 48.66 & 53.93 & 52.49 & 54.18 & 52.32 & 49.80 & 50.97 & 50.10 & 53.72 & 51.15 & 59.01 & 55.99 & 57.50 \\
$-5 \mathrm{~dB}$ & 78.29 & 82.04 & 80.44 & 79.08 & 79.96 & 80.04 & 80.74 & 79.96 & 80.59 & 80.33 & 84.03 & 81.41 & 82.72 \\
Avg. & 18.68 & 18.60 & 18.51 & 21.85 & 19.41 & 18.02 & 18.21 & 17.58 & 19.45 & 18.32 & 22.42 & 20.68 & 21.55 \\
\hline
\end{tabular}

TABLE 5: Recognition results of the CHEQ compensation technique on the Aurora 2 task under clean-condition training (WER \%).

\begin{tabular}{l|rrrrr|rrrrrrrr}
\hline \multirow{2}{*}{ SNR } & \multicolumn{5}{|c|}{ Set A } & \multicolumn{5}{c}{ Set B } & \multicolumn{2}{c}{ Set C } \\
\cline { 2 - 14 } & Subway & Babble & Car & Exhib. & Avg. & Rest. & Street & Airport & Station & Avg. & Subway & Street & Avg. \\
\hline Clean & 1.54 & 1.39 & 1.40 & 1.60 & 1.48 & 1.54 & 1.39 & 1.40 & 1.60 & 1.48 & 1.38 & 1.54 & 1.46 \\
$20 \mathrm{~dB}$ & 3.56 & 2.75 & 2.45 & 4.07 & 3.21 & 3.50 & 2.78 & 2.65 & 3.05 & 3.00 & 3.19 & 2.57 & 2.88 \\
$15 \mathrm{~dB}$ & 6.14 & 4.84 & 3.67 & 6.08 & 5.18 & 5.00 & 4.66 & 4.77 & 4.91 & 4.84 & 5.50 & 3.99 & 4.75 \\
$10 \mathrm{~dB}$ & 9.73 & 9.79 & 7.37 & 11.72 & 9.65 & 9.21 & 8.71 & 6.98 & 8.15 & 8.26 & 10.99 & 9.31 & 10.15 \\
$5 \mathrm{~dB}$ & 18.76 & 20.22 & 16.25 & 22.77 & 19.50 & 20.51 & 18.20 & 17.89 & 17.59 & 18.55 & 22.08 & 19.26 & 20.67 \\
$0 \mathrm{~dB}$ & 38.99 & 45.83 & 37.52 & 42.92 & 41.32 & 44.70 & 39.03 & 40.05 & 39.80 & 40.90 & 45.29 & 42.78 & 44.04 \\
$-5 \mathrm{~dB}$ & 71.63 & 76.63 & 70.15 & 70.01 & 72.11 & 77.24 & 70.71 & 70.92 & 71.34 & 72.55 & 75.84 & 74.00 & 74.92 \\
Avg. & 15.44 & 16.69 & 13.45 & 17.51 & 15.77 & 16.58 & 14.68 & 14.47 & 14.70 & 15.11 & 17.41 & 15.58 & 16.50 \\
\hline
\end{tabular}


TABLE 6: Recognition results of the SE + HEQ compensation technique on the Aurora 2 task under clean-condition training (WER \%).

\begin{tabular}{|c|c|c|c|c|c|c|c|c|c|c|c|c|c|}
\hline \multirow{2}{*}{ SNR } & \multicolumn{5}{|c|}{ Set A } & \multicolumn{5}{|c|}{ Set B } & \multicolumn{3}{|c|}{ Set $C$} \\
\hline & Subway & Babble & Car & Exhib. & Avg. & Rest. & Street & Airport & Station & Avg. & Subway & Street & Avg. \\
\hline$\overline{\text { Clean }}$ & 1.26 & 1.21 & 1.25 & 1.17 & 1.22 & 1.26 & 1.21 & 1.25 & 1.17 & 1.22 & 1.38 & 1.12 & 1.25 \\
\hline $20 \mathrm{~dB}$ & 3.25 & 2.57 & 2.39 & 3.61 & 2.96 & 2.58 & 2.72 & 2.30 & 2.78 & 2.60 & 2.98 & 2.57 & 2.78 \\
\hline $15 \mathrm{~dB}$ & 5.68 & 4.20 & 3.49 & 6.17 & 4.89 & 4.94 & 4.26 & 3.97 & 4.97 & 4.54 & 5.80 & 4.56 & 5.18 \\
\hline $10 \mathrm{~dB}$ & 10.25 & 8.19 & 8.62 & 12.31 & 9.84 & 9.46 & 8.68 & 8.05 & 9.13 & 8.83 & 12.04 & 10.85 & 11.45 \\
\hline $5 \mathrm{~dB}$ & 21.15 & 21.80 & 20.49 & 26.26 & 22.43 & 22.29 & 20.68 & 20.34 & 21.60 & 21.23 & 27.14 & 25.09 & 26.12 \\
\hline $0 \mathrm{~dB}$ & 45.96 & 52.48 & 47.57 & 49.77 & 48.95 & 48.63 & 48.22 & 47.93 & 50.60 & 48.85 & 58.70 & 55.17 & 56.94 \\
\hline$-5 \mathrm{~dB}$ & 77.74 & 83.28 & 82.02 & 79.20 & 80.56 & 80.87 & 79.44 & 81.24 & 81.52 & 80.77 & 83.48 & 80.96 & 82.22 \\
\hline Avg. & 17.26 & 17.85 & 16.51 & 19.62 & 17.81 & 17.58 & 16.91 & 16.52 & 17.82 & 17.21 & 21.33 & 19.65 & 20.49 \\
\hline
\end{tabular}

TABLE 7: Recognition results of the SE + CHEQ compensation technique on the Aurora 2 task under clean-condition training (WER \%).

\begin{tabular}{l|rrrrr|rrrrrrrr}
\hline \multirow{2}{*}{ SNR } & \multicolumn{5}{|c|}{ Set A } & \multicolumn{4}{c}{ Set B } & \multicolumn{2}{c}{ Set C } \\
\cline { 2 - 14 } & Subway & Babble & Car & Exhib. & Avg. & Rest. & Street & Airport & Station & Avg. & Subway & Street & Avg. \\
\hline Clean & 1.57 & 1.60 & 1.76 & 1.42 & 1.59 & 1.57 & 1.60 & 1.76 & 1.42 & 1.59 & 1.41 & 1.48 & 1.45 \\
$20 \mathrm{~dB}$ & 3.32 & 2.51 & 2.24 & 3.36 & 2.86 & 2.76 & 2.99 & 2.30 & 2.50 & 2.64 & 2.73 & 2.33 & 2.53 \\
$15 \mathrm{~dB}$ & 5.56 & 4.56 & 2.89 & 5.49 & 4.63 & 5.43 & 4.53 & 3.85 & 4.57 & 4.60 & 5.10 & 3.99 & 4.54 \\
$10 \mathrm{~dB}$ & 9.46 & 7.80 & 6.32 & 10.49 & 8.52 & 8.54 & 8.10 & 6.74 & 7.56 & 7.74 & 9.86 & 8.98 & 9.42 \\
$5 \mathrm{~dB}$ & 18.27 & 17.84 & 15.09 & 22.00 & 18.30 & 19.34 & 16.78 & 16.67 & 16.78 & 17.39 & 21.09 & 18.68 & 19.89 \\
$0 \mathrm{~dB}$ & 37.64 & 43.83 & 35.19 & 42.02 & 39.67 & 43.60 & 38.48 & 37.31 & 38.75 & 39.54 & 46.64 & 41.72 & 44.18 \\
$-5 \mathrm{~dB}$ & 68.77 & 76.51 & 69.82 & 69.58 & 71.17 & 77.40 & 69.89 & 72.00 & 70.60 & 72.47 & 74.61 & 73.88 & 74.25 \\
Avg. & 14.85 & 15.31 & 12.35 & 16.67 & 14.79 & 15.93 & 14.18 & 13.37 & 14.03 & 14.38 & 17.08 & 15.14 & 16.11 \\
\hline
\end{tabular}

\section{CONCLUSION}

As a feature space compensation approach for robust speech recognition, the conventional HEQ technique can be effectively utilized to compensate for the acoustic mismatch between training and test environments. However, the conventional HEQ has two fundamental limitations caused by the mismatch of phonetic class distributions between training and test data and by the nonmonotonic transformation resulted from the acoustic mismatch. In this paper, to deal with these two problems, a class-based HEQ method is proposed, which not only compensates for the acoustic mismatch but also reduces the limitations of the conventional HEQ by dividing reference and test CDFs into sets of multiple classspecific distributions and then equalizing noisy features on the class basis. For higher phonetic classification accuracy as well as more reliable test CDF estimation in CHEQ, a class-tying technique is employed. In addition, to reduce the acoustic mismatch caused by additive noise, the MMSE-LSAbased speech enhancement is added prior to CHEQ. The experimental results showed the effectiveness of CHEQ by producing EERs of $60 \%$ over MFCC and 19\% over the conventional $\mathrm{HEQ}$, respectively. The addition of SE to CHEQ produces an additional improvement by an ERR of $4 \%$ over CHEQ alone. Moreover, the experimental results strongly imply that the nonmonotonic transformation caused by the acoustic mismatch acts as the major limitation of the conventional HEQ.

\section{REFERENCES}

[1] A. Sankar and C.-H. Lee, "A maximum-likelihood approach to stochastic matching for robust speech recognition," IEEE Transactions on Speech and Audio Processing, vol. 4, no. 3, pp. 190-202, 1996.

[2] X. Huang, A. Acero, and H.-W. Hon, Spoken Language Processing: A Guide to Theory, Algorithm, and System Development, Prentice-Hall, Englewood Cliffs, NJ, USA, 2001.

[3] A. E. Rosenberg, C.-H. Lee, and F. K. Soong, "Cepstral channel normalization techniques for HMM-based speaker verification," in Proceedings of the 3rd International Conference on Spoken Language Processing (ICSLP' '94), pp. 1835-1838, Yokohama, Japan, September 1994.

[4] O. Viikki and K. Laurila, "Cepstral domain segmental feature vector normalization for noise robust speech recognition," Speech Communication, vol. 25, no. 1-3, pp. 133-147, 1998.

[5] C. Kermorvant, "A comparison of noise reduction techniques for robust speech recognition," IDIAP Research Report IDIAP-RR 99-10, IDIAP Research Institute, Martigny, Switzerland, July 1999.

[6] N. S. Kim, Y. J. Kim, and H. Kim, "Feature compensation based on soft decision," IEEE Signal Processing Letters, vol. 11, no. 3, pp. 378-381, 2004.

[7] J. Droppo, L. Deng, and A. Acero, "Evaluation of the SPLICE algorithm on the Aurora2 database," in Proceedings of the 7th European Conference on Speech Communication and Technology (EUROSPEECH '01), pp. 217-220, Aalborg, Denmark, September 2001. 
[8] M. J. F. Gales, "Maximum likelihood linear transformations for HMM-based speech recognition," Computer Speech and Language, vol. 12, no. 2, pp. 75-98, 1998.

[9] R. C. Gonzalez and R. E. Woods, Digital Image Processing, Prentice-Hall, Englewood Cliffs, NJ, USA, 2002.

[10] S. Dharanipragada and M. Padmanabhan, "A nonlinear unsupervised adaptation technique for speech recognition," in Proceedings of the 6th International Conference on Spoken Language Processing (ICSLP'00), vol. 4, pp. 556-559, Beijing, China, October 2000.

[11] F. Hilger and H. Ney, "Quantile based histogram equalization for noise robust speech recognition," in Proceedings of the 7th European Conference on Speech Communication and Technology (EUROSPEECH '01), pp. 1135-1138, Aalborg, Denmark, September 2001.

[12] G. Saon and J. M. Huerta, "Improvements to the IBM Aurora 2 multi-condition system," in Proceedings of the 7th International Conference on Spoken Language Processing (ICSLP '02), pp. 469-472, Denver, Colo, USA, September 2002.

[13] S. Molau, F. Hilger, D. Keysers, and H. Ney, "Enhanced histogram normalization in the acoustic feature space," in Proceedings of the 7th International Conference on Spoken Language Processing (ICSLP '02), pp. 1421-1424, Denver, Colo, USA, September 2002.

[14] S. Molau, F. Hilger, and H. Ney, "Feature space normalization in adverse acoustic conditions," in Proceedings of IEEE International Conference on Acoustics, Speech and Signal Processing (ICASSP '03), vol. 1, pp. 656-659, Hong Kong, April 2003.

[15] F. Hilger, Quantile based histogram equalization for noise robust speech recognition, Ph.D. thesis, RWTH, Aachen-University of Technology, Aachen, Germany, 2004.

[16] J. C. Segura, C. Benítez, Á. de La Torre, A. J. Rubio, and J. Ramírez, "Cepstral domain segmental nonlinear feature transformations for robust speech recognition," IEEE Signal Processing Letters, vol. 11, no. 5, pp. 517-520, 2004.

[17] Á. de La Torre, A. M. Peinado, J. C. Segura, J. L. PérezCórdoba, M. C. Benítez, and A. J. Rubio, "Histogram equalization of speech representation for robust speech recognition," IEEE Transactions on Speech and Audio Processing, vol. 13, no. 3, pp. 355-366, 2005.

[18] Y. Suh and H. Kim, "Class-based histogram equalization for robust speech recognition," ETRI Journal, vol. 28, no. 4, pp. 502-505, 2006.

[19] Y. Ephraim and D. Malah, "Speech enhancement using a minimum mean-square error log-spectral amplitude estimator," IEEE Transactions on Acoustics, Speech, and Signal Processing, vol. 33, no. 2, pp. 443-445, 1985.

[20] Y. Ephraim and D. Malah, "Speech enhancement using a minimum mean-square error short-time spectral amplitude estimator," IEEE Transactions on Acoustics, Speech, and Signal Processing, vol. 32, no. 6, pp. 1109-1121, 1984.

[21] H. Kim and R. C. Rose, "Cepstrum-domain acoustic feature compensation based on decomposition of speech and noise for ASR in noisy environments," IEEE Transactions on Speech and Audio Processing, vol. 11, no. 5, pp. 435-446, 2003.

[22] Y. D. Cho, K. Al-Naimi, and A. Kondoz, "Mixed decisionbased noise adaptation for speech enhancement," Electronics Letters, vol. 37, no. 8, pp. 540-542, 2001.

[23] N. S. Kim and J.-H. Chang, "Spectral enhancement based on global soft decision," IEEE Signal Processing Letters, vol. 7, no. 5, pp. 108-110, 2000.

[24] I. Cohen, "Speech enhancement using a noncausal a priori SNR estimator," IEEE Signal Processing Letters, vol. 11, no. 9, pp. 725-728, 2004.
[25] Final draft ETSI ES 202050 V1.1.1, "Speech Processing, Transmission and Quality aspects (STQ); Distributed speech recognition; advanced front-end feature extraction algorithm; compression algorithms," ETSI, June 2002.

[26] S. S. Chen and R. A. Gopinath, "Gaussianization," in Proceedings of Advances in Neural Information Processing Systems (NIPS '00), pp. 423-429, Denver, Colo, USA, December 2000.

[27] G. Saon, S. Dharanipragada, and D. Povey, "Feature space gaussianization," in Proceedings of IEEE International Conference on Acoustics, Speech and Signal Processing (ICASSP '04), vol. 1, pp. 329-332, Montreal, Que, Canada, May 2004.

[28] C. Nadeu, D. Macho, and J. Hernando, "Time and frequency filtering of filter-bank energies for robust HMM speech recognition," Speech Communication, vol. 34, no. 1-2, pp. 93-114, 2001.

Youngjoo Suh was born in Korea in 1969 and received the B.S. and M.S. degrees in electronics engineering from Kyungpook National University, Korea, in 1991 and 1993, respectively. He received the Ph.D. degree from the School of Engineering, Information and Communications University, Korea, in 2006. From 1993 to 1998, he was a Researcher in the Spoken Language Processing Lab. at the Electronics and Telecommu-

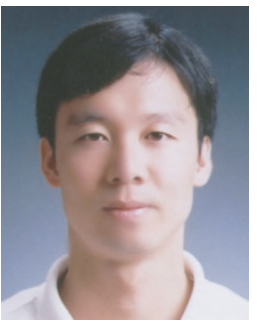
nications Research Institute (ETRI), Korea. In 1999, he served as an Invited Professor at Yeungjin College, Korea. From 2000 to 2002, he worked as a Team Manager at Corevoice Inc., Korea. Since September 2006, he has been a Postdoctoral Researcher at Information and Communications University. His research interests include robust speech recognition and speech enhancement.

Sungtak Kim received the B.S. degree in electronics engineering from Ulsan University and the M.S. degree in multimedia communications and processing from Information and Communications University, Korea, in 2000 and 2003, respectively. He is currently pursuing the Ph.D. degree in multimedia communications and processing at Information and Communications University. His research interests are robust speech recognition and speaker recognition.

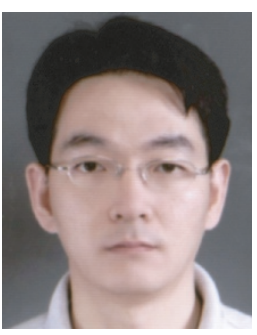

Hoirin Kim was born in Seoul, Korea, in 1961. He received the M.S. and Ph.D. degrees from the Department of Electrical and Electronics Engineering, KAIST, Korea, in 1987 and 1992, respectively. From October 1987 to December 1999, he has been a Senior Researcher in the Spoken Language Processing Lab. at the Electronics and Telecommunications Research Institute (ETRI). From June 1994 to May 1995, he

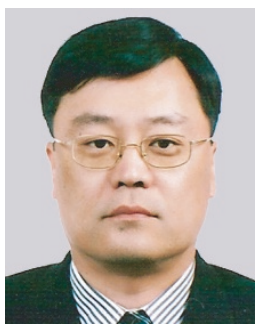
was on leave to the ATR-ITL, Kyoto, Japan. Since January 2000, he is an Associative Professor at Information and Communications University (ICU), Korea. His research interests are signal processing for speech and speaker recognition, audio indexing and retrieval, and spoken language processing. 